\title{
Master Randomization List
}

National Cancer Institute

\section{Source}

National Cancer Institute. Master Randomization List. NCI Thesaurus. Code C115533.

Documentation of the method used to assign individuals to clinical trials. 